\title{
Variation in Capacitance with different Conditions of Sensor
}

\author{
Seema Paliwal \\ Assistant professor \\ EC Department \\ Shri Dadaji Institute of Technology \& Science, \\ Khandwa
}

\begin{abstract}
The projected capacitive sensor is very significant technology for the touch screen in smart phones, tablets and computers Touch is an integral part of the human experience. Capacitive sensing is a technology based on capacitive coupling which takes the capacitance produced by the human body as the input. The Main advantages that capacitive sensing has over other detection approaches are that it can sense different kinds of materials (skin, plastic, metal, liquid), it is contactless and wear-free, it has the ability to sense up to a large distance with small sensor sizes, the PCB Sensor is low cost, and it is a lowpower solution.
\end{abstract}

\section{Keywords}

Capacitive sensor, Dielectric, capacitance, Equilibrium

\section{INTRODUCTION}

Capacitive sensors detect anything that is conductive or has a dielectric different from that of air. Capacitive sensors work by detecting the change of capacitance due to the influence of external objects. The most amazing feature of capacitive sensor is its ability to sense through completely shield housing. Capacitive sensors comprise of electronic circuits that measures capacitance across electrodes also known as antennas.

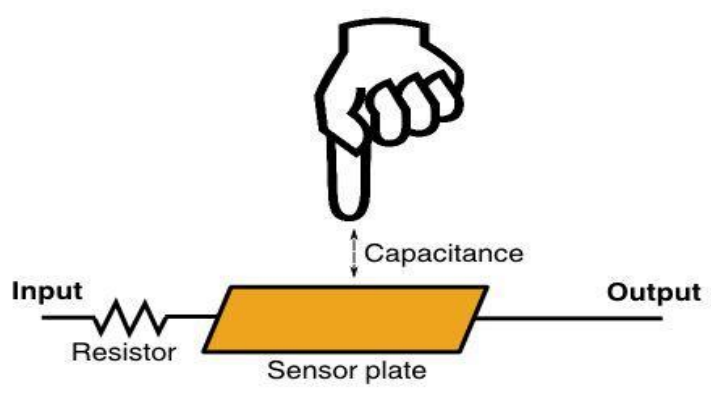

Figure 1: Human Finger with Sensor Plate

\section{EFFECT OF EXTERNAL OBJECT ON CAPACITANCE}

Effect of external object on capacitance:-When conductors are connected to the source the electric field from the source pushes the charges out to the conductors. The positive charge $\&$ negative charge are attractive so they move to each other as close as possible. Charges on the same place are repulsive so they push each other away to the edges. Small charges joined the plate they build up electric field to oppose the others from joining in. Eventually the net force along the conductor is zero and the charges stay in equilibrium.

\author{
Jyoti Krayla \\ Under graduate Student \\ EC Department \\ Shri Dadaji Institute of Technology \& Science, \\ Khandwa
}

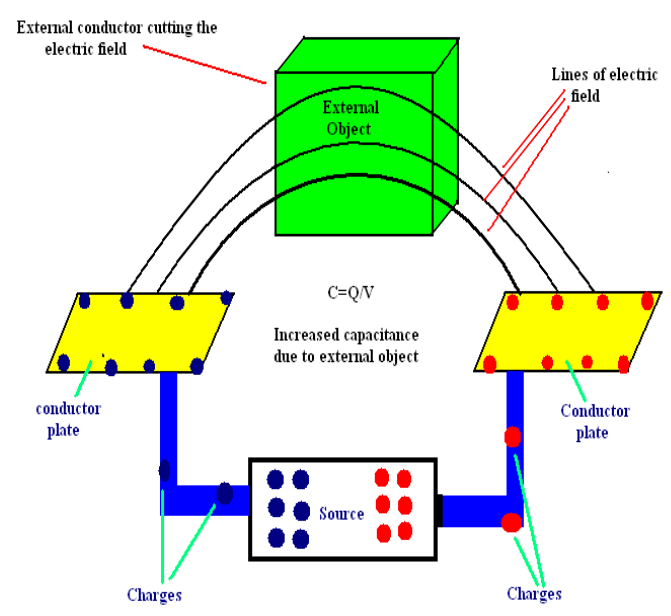

Figure-2 Effect of external object on capacitance

This is when the plates have the same potential difference as the voltage source. "And the capacitance is defined as the ability to store the charges". When the external conductor is put nearby it cuts into the electric field. The electric field polarizes the conductor and the energy is transferred to the polarized charges. Polarized charges get energy from the plates and the plates now have a lower potential. If the source is still connected, more charges will join in the plate and the plates potential is back again. The external object allows more charges to store in the plates \& therefore it increases the capacitance. So even when the external object is not physically connected to any of the plates it is too capable of influencing the capacitance. When the capacitance changes the circuit indicates the presence of an external object. Capacitance increases when external plates are placed nearby. And external plates are polarized by electric field. Two plates are required to detect the nearby object.

\section{THE FUNDAMENTAL PRINCIPLE OF CAPACITIVE TOUCH SENSING}

A common model is studied where electrode forms the one plate of capacitor and the grounded finger forms another plate and changes the overall capacitance of the sensor. In most sensors design one antenna is made touch focus point where another is integrated to the ground plane .The ground plane can pick up the object influence as well. Sensitivity of one plate is increase and sensitivity of other plate reduce by making one antenna more accessible than the other. Another method is to control the size of the antenna. In a simulation to a finger on \& above the coplanar structure the capacitance increases by $12.4 \%$. When the human body is included in the calculation the capacitance increases by $21.7 \%$. The human 
body itself doubles the influence on the capacitance. The body by its huge surface area and finger by its close proximity couples themselves to antennas. If one of the plates is enlarged then the finger approaching the smaller plate becomes the deciding factor for the change of capacitance. If the finger approaches to big plate the capacitance change is minimum $(0.846 \%)$ up to $5.8 \%$. By having large ground plane which is one of the antennas the sensitivity automatically goes to the smaller antenna which is meant to be the touch focus point.

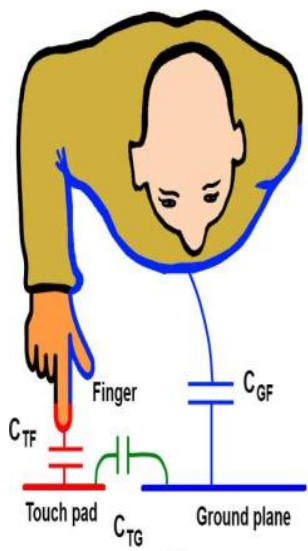

(a)

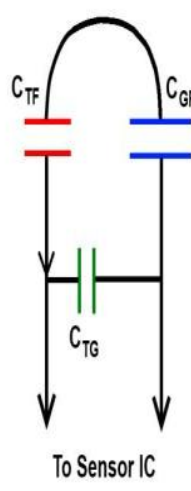

(b)

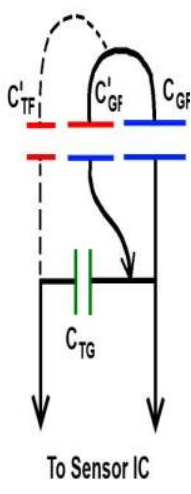

(c)
Figure-3 Illustration of capacitive sensing by human finger

The smaller antenna gains more sensitivity because of high percentage of change compared to the bigger antenna in the same configuration. Even if the ground plate is made smaller and touch antenna may bigger the sensitivity follows the smaller plates. But the smaller antenna do not necessarily get more absolute delta $\mathrm{C}$ than a bigger but less sensitive antenna in another configuration. So to enjoy higher sensitivity from a higher percentage change of smaller antenna the sensing IC must have good signal to noise ratio to begin with.

\section{TECHNOLOGY}

Capacitive touch screens are made up of transparent electrodes arranged in rows and columns. The technology of projected capacitive sensors evolves from its predecessors the self (surface) capacitive sensors. In the self capacitive sensors the touch electrode and the finger tip performs parallel plate capacitors. As the gap $h$ between them closes up the capacitance increases according one over $h$. The charges on electrode which are proportional to the capacitance can be measured to infer the presence of external object.

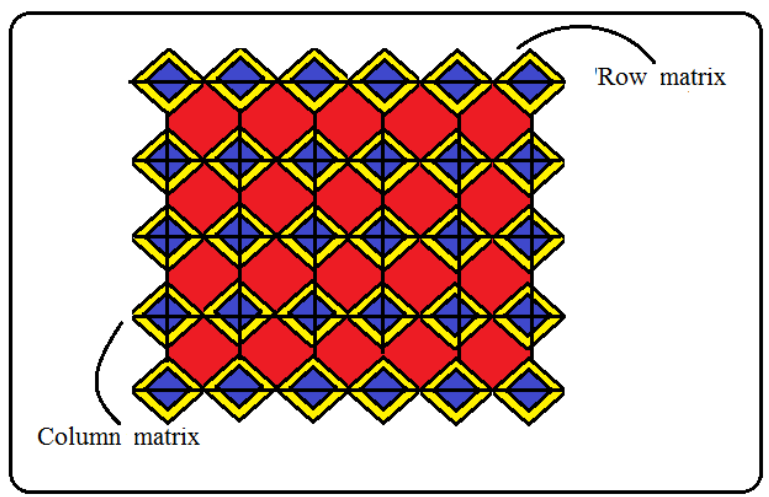

Figure-4 Row and column arrangement of antennas in sensor
Like the mechanical key matrix switch which uses row and column scanning to locate the switch which seen pressed. The capacitive sensors uses the same method for the sensing and this increase the number of sensing channels with the least $\mathrm{I} / \mathrm{O}$ pins

The matrix scanning requires the rows and columns to be electrically isolated and so as one electrode is used as transmitter the electrode is placed next to it without physical contact to sense the influence from the finger. When the transmitter is excited with fixed the voltage the charges induced on the receiver will be affected by the external object. The touch quantity is regarded as mutual capacitance Mc.

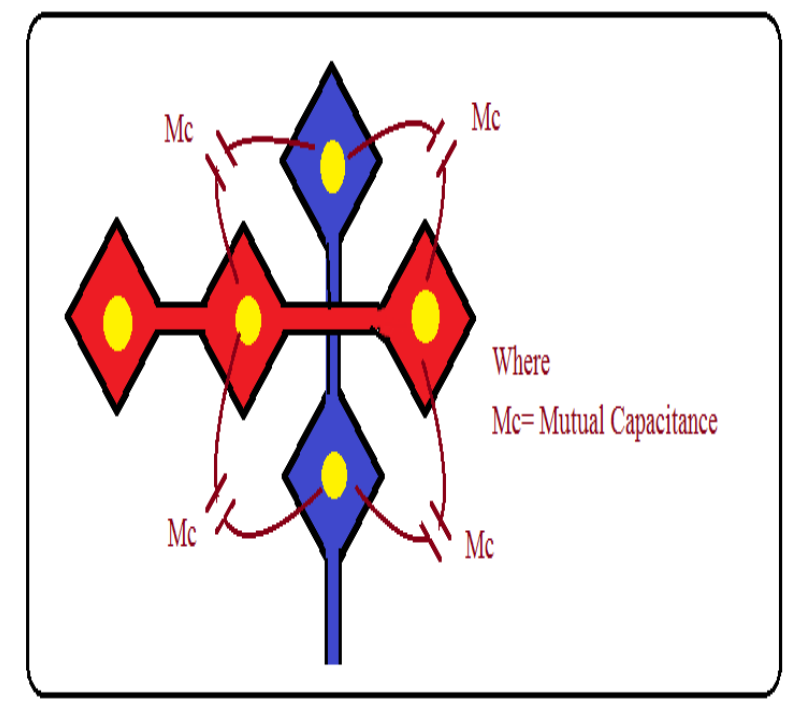

Figure-5 Mutual capacitance illustration

\section{CHARACTERESTICS OF \\ CAPACITIVE SENSORS}

(1) Simple structure, strong adaptability and capacitive sensor structure is simple, easy to manufacture high precision, capacitive sensors are generally made with a metal electrode, inorganic materials for insulation support, so you can work in the high and low temperature, strong radiation and strong magnetic field and other harsh environments, can withstand large temperature changes, under high pressure, high impact, overload, etc.; can measure the difference between high pressure and low pressure.

(2)Dynamic response capacitive sensors as electrostatic attraction between the plates is small, the role of the energy needs of small, moving parts can be made small and thin, light weight, so the high natural frequency, dynamic response time is short, can In the work of a few $\mathrm{MHz}$ frequency, special suitable for dynamic measurement; can use high frequency power supply, so the system operating frequency is high. It can be used to measure the rapidly changing parameters, such as vibration.

(3) High resolution sensor is charged because of gravity between the plates is extremely small, low input energy needs, it is particularly suited to solve the problem of low input energy, such as measuring very small pressure, force and acceleration is very small, displacement can be made very sensitive, very high resolution, can feel $0.001 \mathrm{~m}$, or even smaller displacement.

(4)Temperature stability of capacitance capacitive sensor generally has nothing to do with the electrode materials is conducive to choose materials with low temperature 
coefficient, but also because of their minimal heat, thus affecting the stability is extremely small.

Also figure 7 shows the mutual capacitance begins with some initial value $(\mathrm{Cm} 0)$, decreases slowly hits the maximum deep turns around and then shoots up suddenly. So now it is the DIP not the increase in capacitance that is used for inferring the touch. The turnaround infer the finger moving away and off course there is force detection

\section{RESULT AND CONCLUSION}

Table-1 Variation in capacitance with different conditions of antennas

\begin{tabular}{|c|l|l|l|l|}
\hline Condition & Before & After & $\begin{array}{l}\text { Capacitance } \\
\text { change }\end{array}$ & $\begin{array}{l}\text { Percent } \\
\text { change }\end{array}$ \\
\hline $\begin{array}{c}\text { Small } \\
\text { antennas }\end{array}$ & $0.189 \mathrm{pF}$ & $0.245 \mathrm{pf}$ & $0.056 \mathrm{pF}$ & $30 \%$ \\
\hline $\begin{array}{l}\text { Large } \\
\text { antennas }\end{array}$ & $0.433 \mathrm{pF}$ & $0.594 \mathrm{pF}$ & $0.161 \mathrm{pF}$ & $37 \%$ \\
\hline $\begin{array}{c}\text { Antennas } \\
\text { at short } \\
\text { distance }\end{array}$ & $0.613 \mathrm{Pf}$ & $0.746 \mathrm{pF}$ & $0.133 \mathrm{pF}$ & $22 \%$ \\
\hline
\end{tabular}

Table 1 shows two antennas which are same size then the capacitance increases to $0.056 \mathrm{pF} \&$ there is $30 \%$ change.

- If the antennas are enlarged then the capacitance increases more by $0.161 \mathrm{pF}$ and there is $37 \%$ of increment in capacitance. so larger antennas are easier for measuring the circuits.

- If the distances between them are shortened the electric field is more confined. Although the capacitance is higher but the delta change of capacitance is actually less and make it a less effective sensor.

Figure 6 and figure 7 shows matrix scanning requires the rows and columns to be electrically isolated and so as one electrode is used as transmitter the electrode is placed next to it without physical contact to sense the influence from the finger. When the transmitter is excited with fixed the voltage the charges induced on the receiver will be affected by the external object. The touch quantity is regarded as mutual capacitance $\mathrm{Cm}$. As we know that in parallel plate capacitor the capacitance increases according one over $h$ but experiments has shown that the mutual capacitance at the receiver behaves totally different.

Also figure 7 shows the mutual capacitance begins with some initial value $(\mathrm{Cm} 0)$, decreases slowly hits the maximum deep turns around and then shoots up suddenly. So now it is the DIP not the increase in capacitance that is used for inferring the touch. The turnaround infers the finger moving away and off course there is force detection.

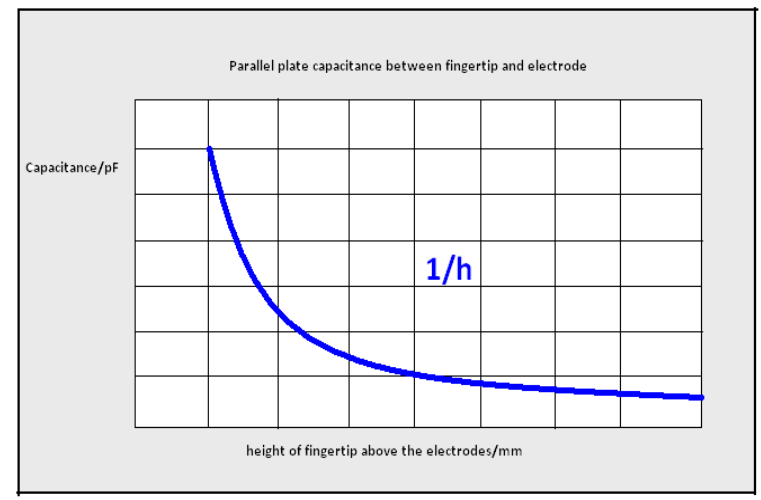

Figure-6 One over $h$ increment in capacitance

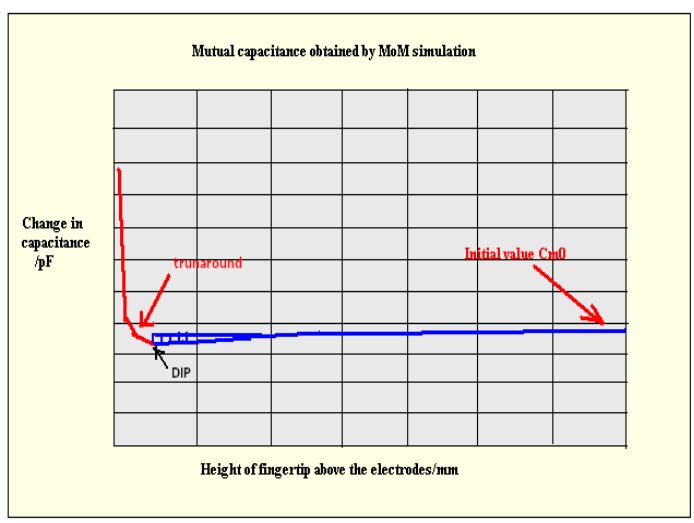

Figure: 7 Decrement of capacitance at initial stage

\section{FUTURE SCOPE}

Capacitive touch sensor will continue to expand to provide manufacturers with more opportunities to incorporate proximity capacitive sensors in their new product designs. Capacitive touch sensing technology has brought new levels of accuracy, usability and reliability in control panel design, and it will continue to help customers create new applications in emerging markets worldwide.

\section{ACKNOWLEDEMENT}

Our special thanks to SDITS college of Khandwa which provide MATLAB Laboratory for this work.

\section{REFERENCES}

[1] A. Kraszewski, S. Trabelsi, S. Nelson, "Moisture Content Determination in Grain by Measuring Microwave Parameters", Measurement Science and Technology, vol. 8, ,pp. 857-863. 1997

[2] D. Wobschall, D. Lakshmanan, "Wireless Soil Moisture Sensor Based on Fringing Capacitance, Proc. of IEEE Sensors 2005, Irvine, California, , , pp. 8-11. October 31November 32005

[3] L. Gradinarsky, H. Brage, B. Lagerholm, I. Björn, S. Folestad, "In Situ Monitoring and Control of Moisture Content in Pharmaceutical Powder Processes Using an Open-Ended Coaxial Probe", Measurement Science and Technology, vol. 17, pp. 1847-1853, 2006.

[4] I. McFarlane, "Control of Final Moisture Content of Food Products Baked in Continuous Tunnel Ovens", Measurement Science and Technology, vol. 17, pp. 241248, 2006. 
[5] Gründler, P. Conductivity sensors and capacitive sensors. In Chemical sensors: An introduction for scientists and engineers (pp. 123-132). Berlin: Springer.(2007)

[6] Ripka, P., \& Tipek, A. Level position and distance. In Modern sensors handbook Newport Beach: ISTE USA. (pp. 305-348) (2007)

[7] A. Fuchs, H. Zangl, G. Holler, "Capacitance-Based Sensing of Material Moisture in Bulk Solids: Applications and Restrictions", Part IV - Capacitive Sensors, Smart Sensors and Sensing Technology in Book Series Lecture Notes in Electrical Engineering, SpringerVerlag, pp. 235-248, 2008.

[8] T. Bretterklieber, H. Zangl, M. Motz, T. Werth, D. Hammerschmidt, "Versatile Sensor Front End for Low-
Depth Modulation Capacitive Sensors", Proc. of the IEEE International Instrumentation and Measurement Technology Conference, Vancouver Island, Canada, May $12-15,2008$, pp. 830-835. INTERNATIONAL JOURNAL ON SMART SENSING AND INTELLIGENT SYSTEMS, VOL. 2, NO. 2, JUNE 2009.

[9] A. Fuchs, M.J. Moser, H. Zangl, T. Bretterklieber, Investigations on the Impact of Different Carrier Frequencies for Capacitance Based Moisture Content Determination in Bulk Solids, to be published in Proc. of the 6th International Conference for Conveying and Handling of Particulate Solids, Brisbane, Australia, August 3-7, 2009. 